\title{
A Quantile Approach to the Interval Shannon Entropy
}

\author{
M. Khorashadizadeh \\ University of Birjand \\ Received: 10/31/2018 Approved: 12/8/2019
}

\begin{abstract}
In this paper, we introduce and study quantile version of the Shannon entropy function via doubly truncated (interval) lifetime, which includes the residual and past lifetimes as special case. We aim to study the use of proposed measure in characterization of distribution functions. Further, we describe a stochastic order and a weighted distribution based on this entropy and show their properties. Finally, some results have been obtained for some distributions such as Uniform, Exponential, Pareto I, Power function and Govindarajulu. Also by analysing a real data the subject has been illustrated.
\end{abstract}

Keywords. Shannon entropy, quantile function, generalized failure rate, quantile doubly truncated Shannon entropy.

MSC 2010: 94A17; 62E10.

\section{Introduction}

Let $X$ be a non-negative absolutely continuous random variable representing the lifetime of a component, then if the component is still operating at time $t$, the residual lifetime is represented by random variable, $X_{t}=X-t \mid X \geqslant t$, and if the component is found to be 'down' at time $t$, the time elapsed from its failure to time $t$ is denoted by random variable $X_{t}^{*}=t-X \mid X \leqslant t$ and has called inactivity or past lifetime. 
Also due to generalizing the two previous situations if we know further information about lower or upper bounds of lifetime of component then we have doubly truncated (interval) random variables of forms $X_{t_{1}, t_{2}}=X-t_{1} \mid t_{1} \leqslant$ $X \leqslant t_{2}$ and $X_{t_{1}, t_{2}}^{*}=t_{2}-X \mid t_{1} \leqslant X \leqslant t_{2}$ where $\left(t_{1}, t_{2}\right) \in D^{*}=\left\{\left(t_{1}, t_{2}\right)\right.$ : $\left.F\left(t_{1}\right)<F\left(t_{2}\right)\right\}$. The random variable $X_{t_{1}, t_{2}}$ is used when the component is still working at time $t_{1}$ and we know that it will not operating greater than time $t_{2}$ and in dual case for random variable $X_{t_{1}, t_{2}}^{*}$ it is used when the component is not working at time $t_{1}$ and we know that the failure has accured in interval $\left(t_{1}, t_{2}\right)$. For various results on doubly truncated random variable in reliability and information theory, we refer to Sankaran and Sunoj (2004), Khorashadizadeh et al. (2013), Kayal and Moharana (2016), Kundu (2017) and Kumar et al. (2019).

As it is well known in information theory, the Shannon entropy (Shannon, 1948) and its dynamic versions includes residual entropy (Ebrahimi, 1996), past entropy (Di Crescenzo and Longobardi, 2002) and interval entropy (Misagh and Yari, 2010, 2011, 2012) are the main measures of the uncertainty contained in random variables $X$, residual lifetime, past lifetime and doubly truncated lifetime which are respectively defined by

$$
\begin{aligned}
H_{X} & =-\int_{0}^{\infty} f(x) \log f(x) d x \\
R E_{X}(t) & =-\int_{t}^{\infty} \frac{f(x)}{\bar{F}(t)} \log \frac{f(x)}{\bar{F}(t)} d x \\
P E_{X}(t) & =-\int_{0}^{t} \frac{f(x)}{F(t)} \log \frac{f(x)}{F(t)} d x \\
H_{X}\left(t_{1}, t_{2}\right) & =-\int_{t_{1}}^{t_{2}} \frac{f(x)}{F\left(t_{2}\right)-F\left(t_{1}\right)} \log \frac{f(x)}{F\left(t_{2}\right)-F\left(t_{1}\right)} d x,
\end{aligned}
$$

where $\left(t_{1}, t_{2}\right) \in D^{*}$ and $H_{X}(0, \infty)$ is the Shannon entropy and $H_{X}(t, \infty)$ is the residual entropy $\left(R E_{X}(t)\right)$ and also $H_{X}(0, t)$ is the past entropy $\left(P E_{X}(t)\right)$. Recently, many researchers have pay attention to use of quantile function (QF) instead of distribution function, which is very usefull in data analysis in applied statistics and is defined for any random variables by,

$$
Q(p)=F^{-1}(p)=\inf \{x \mid F(x) \geqslant p\}, \quad 0 \leqslant p \leqslant 1 .
$$

Quantile functions with respect to distribution functions are less influenced by extreme observation. On the other hand, some important lifetime dis- 
tributions in reliability are known by their quantile functions like Lambda distributions, Power-Pareto distribution and Govindarajulu distribution, so using quantile version of reliability and information measures is the best way to analysis the data for such distributions.

For more properties and usefulness of QF in reliability and information theories we refer readers to the books by Gilchrist (2000) and Nair et al. (2013) and the works by Midhu et al. (2013), Nanda et al. (2014), Sankaran et al. (2016), Belzunce and Martinez-Riquelme (2017), Kundu and Patra (2018), Sankaran and Sunoj (2017), Baratpour and Khammar (2018), Kumar (2018) and Kumar et al. (2019).

From now on, assume that $F$ is continuous, so, $F(Q(u))=F Q(u)=$ $u$. Parzen (1979) have defined the density quantile function with $f Q(u)=$ $f(Q(u))$ and quantile density function with $q(u)=\frac{\partial Q(u)}{\partial u}$, hence $q(u) f Q(u)=$ 1 .

From the perspective of quantile function, Sunoj and Sankaran (2012) introduced a quantile based Shannon entropy and residual entropy $R E_{q}(u)$ respectively by,

$$
H_{q}=\int_{0}^{1} \log q(u) d u
$$

and

$$
R E_{q}(u)=\log (1-u)+(1-u)^{-1} \int_{u}^{1} \log q(p) d p .
$$

They showed that, contrary to the residual entropy of form (1), $R E_{q}(u)$ can uniquely determine the quantile function. Also, Qiu (2018) have obtained more results of (2). Similarly, Sunoj et al. (2013) have studied the past entropy in terms of QF by,

$$
P E_{q}(u)=\log (u)+u^{-1} \int_{0}^{u} \log q(p) d p .
$$

In this paper we define the quantile version of the doubly truncated (interval) Shannon entropy and study some of it properties including characterization, stochastic order, weighted cases and real data analysis. 


\section{Interval Quantile based Shannon Entropy}

In doubly truncation, Navarro and Ruiz (1996) have defined the generalized failure rate (GFR) by,

$$
\left\{\begin{array}{l}
h_{1}\left(t_{1}, t_{2}\right)=\frac{f\left(t_{1}\right)}{\bar{F}\left(t_{1}\right)-\bar{F}\left(t_{2}\right)}, \\
h_{2}\left(t_{1}, t_{2}\right)=\frac{f\left(t_{2}\right)}{F\left(t_{2}\right)-F\left(t_{1}\right)},
\end{array}\right.
$$

where $\left(t_{1}, t_{2}\right) \in D^{*}$. So, in this section, in order to generalize the measures (2) and (3), we first define the quantile GFR by,

$$
\left\{\begin{array}{l}
\Lambda_{1}\left(u_{1}, u_{2}\right)=\frac{1}{\left(u_{2}-u_{1}\right) q\left(u_{1}\right)}, \\
\Lambda_{2}\left(u_{1}, u_{2}\right)=\frac{1}{\left(u_{2}-u_{1}\right) q\left(u_{2}\right)},
\end{array}\right.
$$

where $\left(u_{1}, u_{2}\right) \in D=\left\{\left(u_{1}, u_{2}\right) ; Q\left(u_{1}\right)<Q\left(u_{2}\right)\right\}$.

Although given both $h_{1}\left(t_{1}, t_{2}\right)$ and $h_{2}\left(t_{1}, t_{2}\right)$ the distribution function can be characterized (Navarro and Ruiz, 1996), but given one of $\Lambda_{1}\left(u_{1}, u_{2}\right)$ or $\Lambda_{2}\left(u_{1}, u_{2}\right)$ the quantile density function is uniquely determined for fixed $u \in(0,1)$ by $q(p)=\left[(u-p) \Lambda_{1}(p, u)\right]^{-1}$, for $(p, u) \in D$.

In similar way of previous measures, the doubly truncated (interval) quantile Shannon entropy function is defined by,

$$
\begin{aligned}
H_{q}\left(u_{1}, u_{2}\right) & =-\int_{u_{1}}^{u_{2}} \frac{f(Q(p))}{u_{2}-u_{1}} \log \frac{f(Q(p))}{u_{2}-u_{1}} d Q(p) \\
& =\log \left(u_{2}-u_{1}\right)+\frac{1}{u_{2}-u_{1}} \int_{u_{1}}^{u_{2}} \log (q(p)) d p \\
& =1-\frac{1}{u_{2}-u_{1}} \int_{u_{1}}^{u_{2}} \log \left(\Lambda_{1}\left(p, u_{2}\right) d p\right. \\
& =1-\frac{1}{u_{2}-u_{1}} \int_{u_{1}}^{u_{2}} \log \left(\Lambda_{2}\left(u_{1}, p\right) d p\right.
\end{aligned}
$$

where $\left(u_{1}, u_{2}\right) \in D$.

Definition 1. The random variable $X$ is said to have decreasing (increasing) interval quantile entropy DIQE (IIQE) property in terms of $u_{i}$ if and only if for any fixed $u_{j}, H_{q}\left(u_{1}, u_{2}\right)$ is decreasing (increasing) with respect to $u_{i}$, where $i, j=1,2, i \neq j$ and $\left(u_{1}, u_{2}\right) \in D$. 
Now, differentiating Eq.(4) with respect $u_{1}$, we get

$$
\begin{aligned}
\frac{\partial H_{q}\left(u_{1}, u_{2}\right)}{\partial u_{1}} & =\frac{-1}{u_{2}-u_{1}}+\frac{1}{\left(u_{2}-u_{1}\right)^{2}} \int_{u_{1}}^{u_{2}} \log q(p) d p-\frac{1}{u_{2}-u_{1}} \log q\left(u_{1}\right) \\
& =\frac{1}{u_{2}-u_{1}}\left\{H_{q}\left(u_{1}, u_{2}\right)-\log \left(u_{2}-u_{1}\right)-\log q\left(u_{1}\right)-1\right\} \\
& =\frac{1}{u_{2}-u_{1}}\left\{H_{q}\left(u_{1}, u_{2}\right)+\log \Lambda_{1}\left(u_{1}, u_{2}\right)-1\right\} .
\end{aligned}
$$

So, $H_{q}\left(u_{1}, u_{2}\right)$ is increasing with respect to $u_{1}$ if and only if, for all $\left(u_{1}, u_{2}\right) \in$ $D$,

$$
H_{q}\left(u_{1}, u_{2}\right) \geqslant \log \left(u_{2}-u_{1}\right)+\log q\left(u_{1}\right)+1,
$$

or equivalently

$$
H_{q}\left(u_{1}, u_{2}\right) \geqslant-\log \Lambda_{1}\left(u_{1}, u_{2}\right)+1 .
$$

Similarly differentiating with respect to $u_{2}$ we have,

$$
\begin{aligned}
\frac{\partial H_{q}\left(u_{1}, u_{2}\right)}{\partial u_{2}} & =\frac{1}{u_{2}-u_{1}}-\frac{1}{\left(u_{2}-u_{1}\right)^{2}} \int_{u_{1}}^{u_{2}} \log q(p) d p+\frac{1}{u_{2}-u_{1}} \log q\left(u_{2}\right) \\
& =\frac{1}{u_{2}-u_{1}}\left\{-H_{q}\left(u_{1}, u_{2}\right)+\log \left(u_{2}-u_{1}\right)+\log q\left(u_{2}\right)+1\right\} \\
& =\frac{1}{u_{2}-u_{1}}\left\{-H_{q}\left(u_{1}, u_{2}\right)-\log \Lambda_{2}\left(u_{1}, u_{2}\right)+1\right\} .
\end{aligned}
$$

Hence, $H_{q}\left(u_{1}, u_{2}\right)$ is increasing with respect to $u_{2}$ if and only if, for all $\left(u_{1}, u_{2}\right) \in D$,

$$
H_{q}\left(u_{1}, u_{2}\right) \leqslant \log \left(u_{2}-u_{1}\right)+\log q\left(u_{2}\right)+1,
$$

or equivalently

$$
H_{q}\left(u_{1}, u_{2}\right) \leqslant-\log \Lambda_{2}\left(u_{1}, u_{2}\right)+1 .
$$




\section{Examples}

In this section the interval quantile Shannon entropy for some distributions are obtained.

- Exponential distribution

If $X$ have the Exponential distribution with parameter $\lambda$. Then $Q(p)=$ $\frac{1}{\lambda}(-\log (1-p)), q(p)=\frac{1}{\lambda(1-p)}$ and the interval quantile Shannon entropy is,

$$
\begin{aligned}
H_{q}\left(u_{1}, u_{2}\right)= & \log \left(u_{2}-u_{1}\right)-\frac{1}{u_{2}-u_{1}} \int_{u_{1}}^{u_{2}} \log (\lambda(1-p)) d p \\
= & \log \left(u_{2}-u_{1}\right)-\log \lambda-\frac{\left(1-u_{1}\right)}{\left(u_{2}-u_{1}\right)} \log \left(1-u_{1}\right) \\
& -\frac{\left(u_{2}-1\right)}{\left(u_{2}-u_{1}\right)} \log \left(1-u_{2}\right)+1 .
\end{aligned}
$$

In special case of $u_{1}=u$ and $u_{2}=1$ we have quantile residual entropy,

$$
R E_{q}(u)=1-\log \lambda,
$$

and when $u_{1}=0$ and $u_{2}=u$ we have quantile past entropy,

$$
P E_{q}(u)=1-\log \lambda+\log u+\left(\frac{1-u}{u}\right) \log (1-u) .
$$

- Pareto I distribution

Let $X$ have the Pareto I distribution with $Q(p)=\sigma(1-p)^{-\frac{1}{\alpha}}$ and $q(p)=\frac{\sigma}{\alpha}(1-p)^{-1-\frac{1}{\alpha}}$. Also, we have

$$
\begin{aligned}
H_{q}\left(u_{1}, u_{2}\right)= & \log \left(u_{2}-u_{1}\right)+\frac{1}{u_{2}-u_{1}}\left\{\log \left(\frac{\sigma}{\alpha}\left(u_{2}-u_{1}\right)\right)\right. \\
& +\int_{u_{1}}^{u_{2}}\left(-1-\frac{1}{\alpha}\right) \log (1-p) d p \\
= & K+\log \left(u_{2}-u_{1}\right)-\frac{(\alpha+1)}{\alpha\left(u_{2}-u_{1}\right)} \log \frac{\left(1-u_{1}\right)^{1-u_{1}}}{\left(1-u_{2}\right)^{1-u_{2}}},
\end{aligned}
$$




$$
\begin{aligned}
& R E_{q}(u)=K-\log (1-u)^{\frac{1}{\alpha}}, \\
& P E_{q}(u)=K+\log u(1-u)^{\left(1+\frac{1}{\alpha}\right)\left(\frac{1}{u}-1\right)},
\end{aligned}
$$

where $K=\log \frac{\sigma}{\alpha}+\frac{\alpha+1}{\alpha}$.

- Uniform distribution

Let $X$ have the Uniform distribution with parameters $(a, b)$ and $Q(p)=$ $a+(b-a) p$ and $q(p)=b-a$. Then quantile doubly truncated Shannon entropy for this distribution is,

$$
H_{q}\left(u_{1}, u_{2}\right)=\log \left(u_{2}-u_{1}\right)+\log (b-a) .
$$

In special case of $u_{1}=u$ and $u_{2}=1$ we have quantile residual entropy,

$$
R E_{q}(u)=\log (1-u)+\log (b-a),
$$

and when $u_{1}=0$ and $u_{2}=u$ we have quantile past entropy,

$$
P E_{q}(u)=\log u+\log (b-a) .
$$

- Power function distribution

Let $X$ have the Power function distribution with parameter $(\alpha, \beta)$ and $Q(p)=\alpha p^{\frac{1}{\beta}}$ and $q(p)=\frac{\alpha}{\beta} p^{\frac{1}{\beta}-1}$. Then quantile doubly truncated Shannon entropy for this distribution is given by,

$$
\begin{aligned}
H_{q}\left(u_{1}, u_{2}\right)= & \log \left(u_{2}-u_{1}\right)+\frac{1}{u_{2}-u_{1}}\left\{\log \left(\frac{\alpha}{\beta}\right)\left(u_{2}-u_{1}\right)\right. \\
& \left.+\int_{u_{1}}^{u_{2}}\left(\frac{1}{\beta}-1\right) \log (p) d p\right\} \\
=K & +\log \left(u_{2}-u_{1}\right)-\frac{\left(\frac{1}{\beta}-1\right)\left(u_{1}\right)}{\left(u_{2}-u_{1}\right)} \log \left(u_{1}\right) \\
& +\frac{\left(\frac{1}{\beta}-1\right)\left(u_{2}\right)}{\left(u_{2}-u_{1}\right)} \log \left(u_{2}\right),
\end{aligned}
$$


where $K=\log \frac{\alpha}{\beta}-\frac{1}{\beta}+1$. In special case of $u_{1}=u$ and $u_{2}=1$ we have quantile residual entropy,

$$
R E_{q}(u)=K+\log (1-u)+\left(\frac{\beta-1}{\beta}\right) \frac{u \log u}{(1-u)},
$$

and when $u_{1}=0$ and $u_{2}=u$ we have quantile past entropy,

$$
P E_{q}(u)=K+\frac{1}{\beta} \log u \text {. }
$$

- Govindarajulu distribution

This distribution have introduced by Govindarajulu (1977) with quantile function of the form

$$
Q(u)=\theta+\alpha\left\{(\beta+1) u^{\beta}-\beta u^{\beta+1}\right\} ; \quad 0 \leqslant u \leqslant 1, \alpha, \beta>0, \theta \in R,
$$

which have not closed analytic form of distribution function. In this case we have,

$$
\begin{aligned}
H_{q}\left(u_{1}, u_{2}\right) & =K+\ln \left(\left(u_{2}-u_{1}\right)\left(\frac{u_{2}^{u_{2}(\beta-1)}\left(1-u_{2}\right)^{u_{2}-1}}{u_{1}^{u_{1}(\beta-1)}\left(1-u_{1}\right)^{u_{1}-1}}\right)^{\frac{1}{u_{2}-u_{1}}}\right), \\
R E_{q}(u) & =K+2 \ln (1-u)-\frac{u(\beta-1)}{1-u} \\
P E_{q}(u) & =K+\ln \left(u^{\beta}(1-u)^{1-\frac{1}{u}}\right)
\end{aligned}
$$

where $K=\ln (\alpha \beta(\beta+1))-\beta$.

\section{Characterization}

The next theorem shows that $H_{q}\left(u_{1}, u_{2}\right)$ can determine the distribution uniquely.

Theorem 1. Let $X$ be a non-negative absolutely continuous random variable then the quantile density function is uniquely determined for fixed $u \in(0,1)$ by interval quantile entropy via relation,

$$
q(p)=\exp \left\{1-\log (u-p)+H_{q}(p, u)-(u-p) H_{q}^{\prime}(p, u)\right\} ; \quad(p, u) \in D .
$$


Proof. The proof follows from (5).

Theorem 2. If $X$ is IIQE (DIQE) in terms of $u_{i}, i=1,2$ and if $\phi($.$) is any$ non-negative, increasing and convex (concave) function, then $\phi(X)$ is also $I I Q E$ (DIQE) in terms of $u_{i}, i=1,2$.

Proof. Let $g(t)$ be the probability density function of $T=\phi(X)$, so $g(t)=$ $\frac{f\left(\phi^{-1}(t)\right)}{\phi^{\prime}\left(\phi^{-1}(t)\right)}$ and hence, $g\left(Q_{T}(u)\right)=\frac{1}{q_{T}(u)}=\frac{f Q(u)}{\phi^{\prime} Q(u)}=\frac{1}{q_{X}(u) \phi^{\prime} Q(u)}$, then the interval quantile Shannon entropy of $T, H_{q}^{T}\left(u_{1}, u_{2}\right)$ is as follows,

$$
\begin{aligned}
H_{q}^{T}\left(u_{1}, u_{2}\right) & =\log \left(u_{2}-u_{1}\right)+\frac{1}{u_{2}-u_{1}} \int_{u_{1}}^{u_{2}} \log \left(q_{T}(p)\right) d p \\
& =\log \left(u_{2}-u_{1}\right)+\frac{1}{u_{2}-u_{1}} \int_{u_{1}}^{u_{2}} \log \left(q_{X}(p) \phi^{\prime}(Q(p))\right) d p \\
& =\log \left(u_{2}-u_{1}\right)+\frac{1}{u_{2}-u_{1}} \int_{u_{1}}^{u_{2}} \log \left(q_{X}(p)\right) d p+\frac{1}{u_{2}-u_{1}} \int_{u_{1}}^{u_{2}} \log \left(\phi^{\prime}(Q(p)) d p\right. \\
& =H_{q}^{X}\left(u_{1}, u_{2}\right)+E\left(\log \left(\phi^{\prime}(X)\right) \mid \phi^{-1}\left(u_{1}\right)<X<\phi^{-1}\left(u_{2}\right)\right),
\end{aligned}
$$

where $\left(u_{1}, u_{2}\right) \in D$ and $H_{q}^{X}\left(u_{1}, u_{2}\right)$ and $H_{q}^{T}\left(u_{1}, u_{2}\right)$ are the interval quantile Shannon entropy of $X$ and $T$ respectively. Now if $X$ is IIQE and $\phi(x)$ is non-negative, increasing and convex then $\phi(x)$ is also IIQE. So the proof is completed.

It should be noted that all characterization problems of distribution functions discussed in literature are hold for quantile version of measures with transformation $x=Q(u)$. But, new useful characterizations can be obtianed in the quantile case. Nair et al. (2013) have obtained characterization results based on linear hazard quantile function and mean residual quantile function.

The following theorems characterize different distributions using $H_{q}\left(u_{1}, u_{2}\right)$, which their proofs are direct.

Theorem 3. The non-negative random variable $X$ have interval quantile Shannon entropy of the form,

$$
H_{q}\left(u_{1}, u_{2}\right)=\frac{1}{a\left(u_{2}-u_{1}\right)} \ln \left(\frac{b^{b}}{\left(a\left(u_{2}-u_{1}\right)+b\right)^{a\left(u_{2}-u_{1}\right)+b}}\right),
$$


if and only if

$$
\Lambda_{i}\left(u_{1}, u_{2}\right)=a\left(u_{2}-u_{1}\right)+b ; \quad i=1,2,
$$

where $\left(u_{1}, u_{2}\right) \in D, a, b>0$.

It can be seen that the Equations (7) and (8) are hold if and only if for fixed $u \in(0,1)$,

$$
\begin{aligned}
q(p) & =\frac{1}{a(u-p)^{2}+b(u-p)} \\
Q(p) & =\frac{1}{b} \ln \left(\frac{u(a(u-p)+b)}{(u-p)(a u+b)}\right) .
\end{aligned}
$$

Corollary 1. In special case of $b=0$ in Theorem 3, the lifetime random variable $X$ have interval quantile Shannon entropy of the form $H_{q}\left(u_{1}, u_{2}\right)=$ $\ln \frac{1}{a\left(u_{2}-u_{1}\right)}$ if and only if $\Lambda_{i}\left(u_{1}, u_{2}\right)=a\left(u_{2}-u_{1}\right) ; i=1,2$ and also, $q(p)=$ $\frac{1}{a(u-p)^{2}}$ and $Q(p)=\frac{p}{a u(u-p)}$.

Theorem 4. The non-negative random variable $X$ have interval quantile Shannon entropy of the form,

$$
H_{q}(p, u)=a \ln (u-p)+b,
$$

if and only if for any fixed $u \in(0,1)$

$$
Q(p)=K\left(u^{a}-(u-p)^{a}\right),
$$

where $(p, u) \in D$ and $K=\frac{e^{a+b+1}}{a}$ is constant.

Theorem 5. A random variable $X$ has quantile density function of the form

$$
q(p)=K p^{\alpha}(u-p)^{-(\gamma+\alpha)},
$$

for any fixed $u \in(0,1)$ and all $(p, u) \in D$ if and only if it satisfies the relationship

$$
H_{q}(p, u)=\log \left(K u^{\frac{a u}{u-p}}(u-p)^{-(\gamma+\alpha)+1}\right),
$$

where $\alpha$ and $\gamma$ are real constants. 
Remark 1. The family of distributions (9) in special case of $u=1$ contains several distributions such as Exponential $(\alpha=0, \gamma=1)$, rescaled Beta $(\alpha=0, \gamma>1)$, Pareto $(\alpha=0, \gamma<1)$, Loglogistic $(\alpha=\lambda-1, \gamma=2)$ and Govindarajulu $(\alpha=\beta-1, \gamma=-\beta)$.

\section{Stochastic Order}

In this section some stochastic order properties of interval quantile Shannon entropy are obtained.

Definition 2. The random variable $X$ is said to have less $I Q E$ than $Y$ if $H_{q}^{Y}\left(u_{1}, u_{2}\right) \geqslant H_{q}^{X}\left(u_{1}, u_{2}\right)$ for all $\left(u_{1}, u_{2}\right) \in D$, and we write $X \leqslant_{I Q E} Y$.

For example if $X$ and $Y$ have Uniform distribution with parameters $(0, a)$ and $(0, b)$ respectively, and if $a<b$, then

$H_{q}^{X}\left(u_{1}, u_{2}\right)=\log \left(u_{2}-u_{1}\right)+\log (a) \leqslant \log \left(u_{2}-u_{1}\right)+\log (b)=H_{q}^{Y}\left(u_{1}, u_{2}\right)$,

so, $X \leqslant_{I Q E} Y$.

Theorem 6. Let $Z_{1}=a_{1} X+b_{1}$ and $Z_{2}=a_{2} Y+b_{2}$, where $a_{1}>0, a_{2}>0$ and $b_{1}>0, b_{2}>0$. If $X \leqslant_{I Q E} Y$ and $a_{1} \leqslant a_{2}$, then $Z_{1} \leqslant_{I Q E} Z_{2}$.

Proof. Using (4), the quantile density function of the variable $Z=a X+b$, where $a>0$ and $b>0$, is of the form,

$$
H_{q}^{Z}\left(u_{1}, u_{2}\right)=H_{q}^{X}\left(u_{1}, u_{2}\right)+\log (a),
$$

which proved the theorem.

\section{Weighted Case}

Fisher (1934) has introduced the concept of weighted distribution, which has many applications in many areas of applied statistics. This concept is related to the case of when our observations are recorded with some weight function like $w(x)>0$. For more properties of weighted distributions and its applications one can see the works by Navarro et al. (2006), Bartoszewicz (2009) and Gupta and Arnold (2016) and references therein. Let $w(x)$ be a nonnegative function of $x$ such that $E(w(X))=\int_{0}^{\infty} w(x) f(x) d x$ is finite. Then 
the corresponding probability density and distribution functions weighted random variable $X^{w}$ are given by

$$
\begin{aligned}
f^{w}(x) & =\frac{w(x) f(x)}{E(w(X))} \\
F^{w}(x) & =\frac{E(w(X) \mid X \leqslant x)}{E(w(X))} F(x) .
\end{aligned}
$$

When the weight function depends only on the length of the unit of interest (i.e. $w(x)=x$ ), the $X^{w} \equiv X^{*}$ is called length-biased or a size-biased random variable with probability density and distribution functions as follow,

$$
\begin{aligned}
f^{*}(x) & =\frac{x f(x)}{E(X)} \\
F^{*}(x) & =E(X)^{-1} \int_{0}^{x} t f(t) d t .
\end{aligned}
$$

The corresponding weighted density and distribution function based on quantile functions can be expressed in the following forms,

$$
\begin{aligned}
f^{w}(Q(u)) & =\frac{w(Q(u)) f(Q(u))}{\mu^{w}} \\
F^{w}(Q(u)) & =\frac{E(w(Q(U)) \mid Q(U) \leqslant Q(u))}{\mu^{w}} u
\end{aligned}
$$

where

$$
\mu^{w}=\int_{0}^{1} w(Q(p)) f(Q(p)) d Q(p)=\int_{0}^{1} w(p) d p<\infty .
$$

Hence, it is easy to see that the weighted quantile density function is $q^{w}(u)=$ $\frac{\mu^{w} q(u)}{w(Q(u))}$. So, the weighted interval quantile Shannon entropy can be defined 
by

$$
\begin{aligned}
H_{q}^{w}\left(u_{1}, u_{2}\right) & =\log \left(u_{2}-u_{1}\right)+\frac{1}{u_{2}-u_{1}} \int_{u_{1}}^{u_{2}} \log \left(q^{w}(p)\right) d p \\
& =H_{q}\left(u_{1}, u_{2}\right)+\log \mu^{w}-\frac{1}{u_{2}-u_{1}} \int_{u_{1}}^{u_{2}} \log w(Q(p)) d p,
\end{aligned}
$$

and in speacial case of length-biased $(w(Q(p))=Q(p))$, we have,

$$
H_{q}^{w}\left(u_{1}, u_{2}\right)=H_{q}\left(u_{1}, u_{2}\right)+\log E_{q}(X)-\frac{1}{u_{2}-u_{1}} \int_{u_{1}}^{u_{2}} \log Q(p) d p .
$$

Theorem 7. If $X$ is IIQE (DIQE) in terms of $u_{i}, i=1,2$ and if $w(x)$ is any non-negative, increasing and convex (concave) function, then $X^{w}$ is also IIQE (DIQE) in terms of $u_{i}, i=1,2$.

Proof. The proof is similar to that of Theorem 2.

Corollary 2. In case of length-biased $(w(x)=x)$, the IIQE (DIQE) properties of $X$, implies the IIQE (DIQE) of $X^{*}$.

\section{Data analysis}

The intended data include the production of gas oil (in cubic meters per day) in Iranian refineries from 1998 to 2014 (extracted from National Statistics Center database) as follows:

$$
\begin{aligned}
& \text { 64731, 69545, 69945, 70879, 71923, 73154, 77037, 79215, 80473, 81549, } \\
& 84957,88702,90951,94677,93595,97689,96016
\end{aligned}
$$

Shokrani and Khorashadizadeh (2019) have shown that these data have Govindarajulu distribution of the form (6) with parameters $\theta=66890.09, \alpha=$ 31052.52 and $\beta=2.26$. For this data, we have computed the quantile interval Shannon entropy as follows,

$$
H_{q}\left(u_{1}, u_{2}\right)=7.79+\ln \left(\left(u_{2}-u_{1}\right)\left(\frac{u_{2}^{1.26 u_{2}}\left(1-u_{2}\right)^{u_{2}-1}}{u_{1}^{1.26 u_{1}}\left(1-u_{1}\right)^{u_{1}-1}}\right)^{\left(u_{2}-u_{1}\right)^{-1}}\right) .
$$




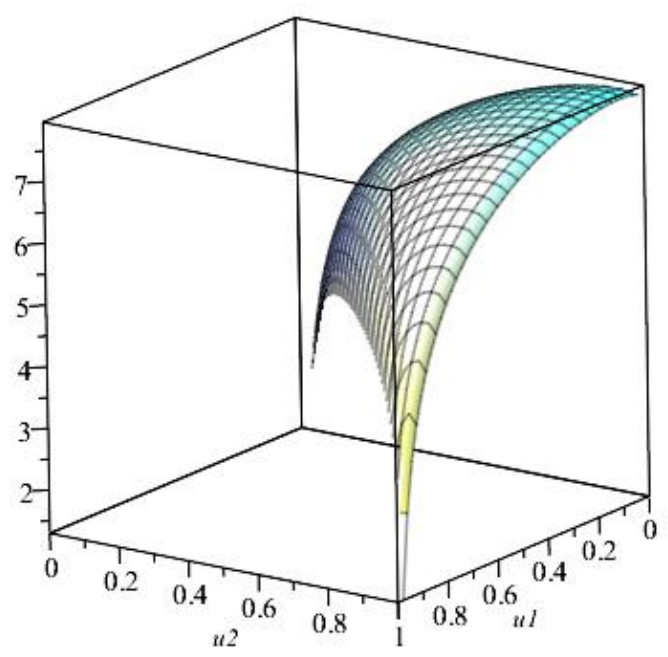

Figure 1. The quantile interval Shannon entropy of the production of gas oil data

The Figure 1 shows the values of $H_{q}\left(u_{1}, u_{2}\right)$ for different values of $\left(u_{1}, u_{2}\right)$, which shows that the maxiumum uncertainty of production of gas oil data is 7.79 .

\section{Acknowledgment}

The authors are grateful to the editors and reviewers of the manuscript.

\section{References}

Baratpour, S. and Khammar, A.H. (2018). A Quantile-based Generalized Dynamic Cumulative Measure of Entropy. Communications in Statistics - Theory and Methods, 47, 3104-3117.

Bartoszewicz, J. (2009). On a Representation of Weighted Distributions. Statistics and Probability Letters, 79, 1690-1694.

Belzunce, F. and Martinez-Riquelme, C. (2017). On Sufficient Conditions for the Comparison of Some Quantile-based Measures. Communications in Statistics - Theory and Methods, 46, $6512-6527$. 
Di Crescenzo, A. and Longobardi, M. (2002). Entropy-based Measure of Uncertainty in Past Lifetime Distribution. J. Appl. Probab., 39, 434-440.

Ebrahimi, N. (1996). How to Measure Uncertainty in the Residual Life Time Distribution. Sankhya: The Indian Journal of Statistics, Series A, 48-56.

Fisher, R.A. (1934). The Effects of Methods of Ascertainment upon the Estimation of Frequencies. Ann. Eugenics, 6, 13-25.

Gilchrist, W. (2000). Statistical Modelling with Quantile Functions. Chapman and Hall/CRC, Boca Raton, FL.

Govindarajulu, A. (1977). A Class of Distributions Useful in Lifetesting and Reliability with Applications to Nonparametric Testing. In: Tsokos, C.P., Shimi, I.N. (Eds.), Theory and Applications of Reliability, Vol. 1. Academic Press, New York, pp. 109-130.

Gupta, R.C. and Arnold, B.C. (2016). Preservation of Failure Rate Function Shape in Weighted Distributions. AStA Advances in Statistical Analysis, 100, 1-20.

Kayal, S. and Moharana, R. (2016). Some Results on a Doubly Truncated Generalized Discrimination Measure. Applications of Mathematics, 61, 585-605.

Khorashadizadeh, M., Rezaei Roknabadi, A.H. and Mohtashami Borzadaran, G.R. (2013). Doubly Truncated (interval) Cumulative Residual Andpast Entropy. Statistics and Probability Letters, 83, 1464-1471.

Kundu, C. (2017). On Weighted Measure of Inaccuracy for Doubly Truncated Random Variables. Communications in Statistics - Theory and Methods, 46, 3135-3147.

Kundu, C. and Patra, A. (2018). Some Results on Residual Life and Inactivity Time at Random Time. Communications in Statistics - Theory and Methods, 47, 372-384.

Kumar, V.R. (2018). A Quantile Approach of Tsallis Entropy for Order Statistics. Physica A: Statistical Mechanics and its Applications, 503, 916-928.

Kumar, V., Taneja, G. and Chhoker, S. (2019). Some Results on Quantile-based Shannon Doubly Truncated Entropy. Statistical Theory and Related Fields, 3, 59-70.

Midhu, N.N., Sankaran, P.G. and Nair, N.U. (2013). A Class of Distributions with the Linear Mean Residual Quantile Function and Its Generalizations. Statistical Methodology, 15, 1-24.

Misagh, F. and Yari, G.H. (2010). A Novel Entropy-based Measure of Uncertainty to Lifetime Distributions Characterizations. In: Proc. ICMS 10. Ref. No. 100196. Sharjah, UAE.

Misagh, F. and Yari, G.H. (2011). On Weighted Interval Entropy. Statistics and Probability Letters, 29, 167-176. 
Misagh, F. and Yari, G.H. (2012). Interval Entropy and Informative Distance. Entropy, 14, 480-490.

Nair, N.U., Sankaran, P.G. and N. Balakrishnon, (2013). Quantile-Based Reliability Analysis. Springer, New York, Heidelberg, Dordrecht, London.

Nanda, A.K., Sankaran, P.G. and Sunoj, S.M. (2014). Residual Renyis Entropy: A Quantile Approach. Statist. Probab. Lett., 85, 114-121.

Navarro, J. and Ruiz, J.M. (1996). Failure-rate Function for Doubly-truncated Random Variables. IEEE Transactions on Reliability, 45, 685-690.

Navarro, J., Ruiz, J.M. and Del Aguila, Y. (2006). Multivariate Weighted Distributions: a Review and Some Extensions. Statistics, 40, 51-64.

Parzen, E. (1979). Nonparametric Statistical Data Modeling. J. Amer. Statist. Assoc., 74, 105-122.

Qiu, G. (2018). Further Results on the Residual Quantile Entropy. Communications in Statistics - Theory and Methods, 47, 3092-3103.

Sankaran, P.G. and Sunoj, S.M. (2004). Identification of Models using Failure Rate and Mean Residual Life of Doubly Truncated Random Variables. Statistical Papers, 45, 97-109.

Sankaran, P.G. and Sunoj, S.M. (2017). Quantile based Cumulative Entropies. Communications in Statistics - Theory and Methods, 46, 805-814.

Sankaran, P.G., Sunoj, S.M. and Nair, N.U. (2016). Kullback-Leibler Divergence: A Quantile Approach. Statistics and Probability Letters, 111, 72-79.

Shannon, C.E., (1948). A Mathematical Theory of Communication. Bell Syst. Tech. J., 27, 379-423.

Shokrani, A. and Khorashadizadeh, M. (2019). Dynamic Quantile Inaccuracy Measure between Two Past Lifetimes. Journal of Statistical Sciences, 12, 449-468.

Sunoj, S.M. and Sankaran, P.G., (2012). Quantile based Entropy Function. Statistics and Probability Letters, 82, 1049-1053.

Sunoj, S.M., Sankaran, P.G. and Nanda, A.K. (2013). Quantile based Entropy Function in Past Lifetime. Statistics and Probability Letters, 83, 366-372. 


\section{Mohammad Khorashadizadeh}

Department of Statistics,

University of Birjand,

Birjand, Iran.

email:m.khorashadizadeh@birjand.ac.ir 